\title{
Minimizing a Wireless Passive LC-Tank Sensor to Monitor Bladder Pressure: A Simulation Study
}

\author{
Jacob Melgaard $^{1}$ (D) $\cdot$ Johannes J. Struijk $^{1} \cdot$ Nico J. M. Rijkhoff $^{1}$
}

Received: 4 March 2016/ Accepted: 22 September 2016/Published online: 16 June 2017

(C) Taiwanese Society of Biomedical Engineering 2017

\begin{abstract}
In this simulation study, a wireless passive LCtank sensor system was characterized. Given the application of continuous bladder monitoring, a specific system was proposed in terms of coil geometries and electronic circuitry. Coupling coefficients were spatially mapped by simulation, as a function of both coil distance, and longitudinal and transverse translation of the sensor relative to the antenna. Further, two interrogation schemes were outlined. One was an auto-balancing bridge for computing the sensor-system impedance. In this case, the theoretical noise limit of the analogue part of the system was found by simulations. As the full system is not necessary for obtaining a pressure reading from the sensor, a simplified circuit more suited for an implantable system was deduced. For this system, both the analogue and digital parts were simulated. First, the required ADC resolution for operating the system at a given coupling was found by simulations in the noise-free case. Then, for one selected typical operational point, noise was added gradually, and through Monte-Carlo type simulations, the system performance was obtained. Combining these results, it was found that it at least is possible to operate the proposed system for distances up to $12 \mathrm{~mm}$, or equivalently for coupling coefficients above 0.005 . In this case a 14 bit $\mathrm{ADC}$ is required, and a carrier SNR of $27 \mathrm{~dB}$ can be tolerated.
\end{abstract}

Keywords Urinary bladder - Closed loop stimulation . Pressure sensor · Wireless sensor

Jacob Melgaard

jm@hst.aau.dk

1 Department of Health Science and Technology, The Faculty of Medicine, Aalborg University, Fredrik Bajers Vej 7, 9220 Aalborg $\varnothing$, Denmark

\section{Introduction}

To make a fully implantable neural prosthesis to treat neurogenic detrusor overactivity (NDO) by conditional stimulation [1], a sensor capable of detecting the onset of urinary bladder contractions is necessary. The basic principle is that the small pressure increases in the beginning involuntary detrusor contractions can be used to detect the onset of these contractions, and prompt stimulation of pudendal afferent nerves can abolish the contractions before leakage occurs. Further details and a discussion of the system has previously been published [2]. Catheterbased pressure monitoring systems for the bladder have been proposed [3-5]. However, there are several disadvantages of this approach. Contact with urine causes encrustation of the catheter or tip membrane $[4,6]$. Coating with certain materials may reduce the rate of encrustation, but there are yet no known permanent solutions to the problem [7]. Another way of circumventing encrustation is placing a catheter-tip transducer on the outer surface of the bladder or in the bladder wall. However, another problem arises from this, and that is the risk of detachment or migration. Koldewijn and colleagues implanted fluid-filled capsules in goats at different sites and with different sutures, and experienced problems with migration in all but one configuration [8]. It is hypothesized that the relative large size (Ø $25 \mathrm{~mm}$, height $3 \mathrm{~mm}$ ), and the rigid tubes connected to them, may have been the primary factors for the migration process. Hence, it is expected that small wireless pressure sensors will perform better with respect to migration.

In biomedical applications, wireless passive LC-tank sensors offer a range of advantages over battery-powered sensors. They are simpler, can be made smaller, and the sensors themselves do not have a limited battery life. The 
disadvantage is that a certain coupling between transceiver antenna and sensor is necessary to ensure adequate modulation of the signal to obtain a sensor reading. A LC-tank sensor is typically a variable capacitor (the gap between the plates varies with pressure) connected to a coil. Other designs are possible, but this is the only design considered in this work. As sensors are miniaturized, the operational distance generally decreases and susceptibility to noise increases, both due to reduced coupling. For a given sensor geometry and operational distance, the antenna coil may be optimized to provide optimal coupling. Sometimes, however, this optimization is constrained by limited space and/ or geometrical limitations, making this task difficult. If, in addition, the sensor moves during operation, this further complicates matters. While the optimization task is specific for each application, some general limitations are determined by the coupling between sensor and antenna, the noise level of the system, the readout scheme and the resolution of the analog to digital converters (ADCs) used in the system. These details are seldomly described; the focus is usually on sensitivity, sensor range optimization or manufacturing technique [9-14].

For sensors intended for bladder monitoring, there is the additional problem of bladder movements. Bladder movements are expected to occur primarily due to the bladder constantly undergoing voiding and filling cycles, but also due to changes in rectal volume and forces exerted by bowels. For a wired sensor placed in the bladder wall, such movements may cause sensor migration, if for instance the wire becomes fixed in connective tissue. The amount of bladder movement has mostly been described in relation to radiotherapy for bladder cancer, however, in this context the focus was on the range of movement and not on the movement path. Still, from these studies it can be inferred that movement is expected to occur along approximately linear paths radiating from the urethra, the only point at which the bladder is fixed. In the lateral view (para-sagittal plane), the largest movements were described in the anterior and cranial direction along a line angled approximately $60-70^{\circ}$ to horizontal $[15,16]$. Movements up to $4 \mathrm{~cm}$ were described in this direction. If the bladder was regarded a sphere fixed at one point at the periphery (i.e. the urethra), the same estimated movement is found for bladder volumes from 30 to $500 \mathrm{ml}$. For robust operation of a LC-tank sensor, a constant coupling is preferred. Based on this, an antenna and sensor design is proposed, that is intended to accommodate a constant coupling over this range of movement.

The aim of this study was threefold. Firstly, to investigate the coupling between a proposed sensor and antenna design intended for monitoring the pressure in the urinary bladder. Secondly, to find the limitations of the system in terms of coupling, SNR and ADC resolution. Finally, to present a simplified interrogation scheme for LC-tank sensors, and to compare the performance to a traditional system, with respect to noise and coupling tolerance.

\section{Methods}

\subsection{System Description}

The proposed system consists of a small square sensor $(6 \times 6 \times 1 \mathrm{~mm})$ and a larger rectangular antenna (measuring $40 \mathrm{~mm}$ by $10 \mathrm{~mm}$ ). The antenna is intended to be placed directly adjacent to the bladder, while the sensor is intended to be placed in the middle of the bladder wall. An illustration of the intended position of the sensor, and the expected movement during filling, is shown in Fig. 1. The sensor should be placed in a pouch in the middle of the bladder wall, while the antenna coil could be anchored to the pubic symphysis. The intention is that during the bladder movements expected during normal operation, the sensor will always be well coupled with the antenna. But contrary to a wired sensor, there is reduced risk of migration or erosion [17].

Electrically, the LC-tank sensor system can be described as a modulated air-coupled transformer (see Fig. 2). The impedance, given in Eq. (1), shows a phase dip at the sensor resonance frequency, which can be used to compute the sensor capacity and thus the sensed pressure.

$Z_{a}=\frac{\boldsymbol{V}_{a}}{\boldsymbol{I}_{a}}=R_{a}+j \omega L_{a}+\frac{\omega^{2} M^{2}}{R_{s}+j \omega L_{s}+\frac{1}{j \omega C_{s}}}$

At sensor resonance, the inductive and capacitive parts of the sensor impedance cancel. Noting further that $M=k \sqrt{L_{s} L_{a}}$, where $0 \leq k \leq 1$ denotes the coupling coefficient, the phase dip at resonance can be obtained:

$$
\begin{aligned}
\angle_{\text {dip }}\left(Z_{a}\left(\omega_{0}\right)\right) & =\operatorname{arccot}\left(\frac{\operatorname{Im}\left(Z_{a}\left(\omega_{0}\right)\right)}{\operatorname{Re}\left(Z_{a}\left(\omega_{0}\right)\right)}\right) \\
& =\arctan \left(\frac{\operatorname{Re}\left(Z_{a}\left(\omega_{0}\right)\right)}{\operatorname{Im}\left(Z_{a}\left(\omega_{0}\right)\right)}\right) \\
& =\arctan \left(\frac{R_{a}}{\omega_{0} L_{a}}+\frac{\omega_{0} k^{2} L_{s}}{R_{s}}\right)
\end{aligned}
$$

Typically, $R_{a} \ll \omega_{0} L_{a}$, so the first tem in the $\arctan$ in (2) can be neglected. It then becomes clear, that the coupling, $k$, is the key parameter for the dip magnitude. If $M$ increases due to increased $L_{a}$, while $k$ remains the same, the dip magnitude does not increase.

To interrogate the sensor, an auto-balancing bridge (ABB) topology can be used. The ABB provides an accurate estimate of the impedance, both magnitude and phase. However, in terms of implantable systems, it is rather complex, requiring four simultaneous ADCs and a 
(a)

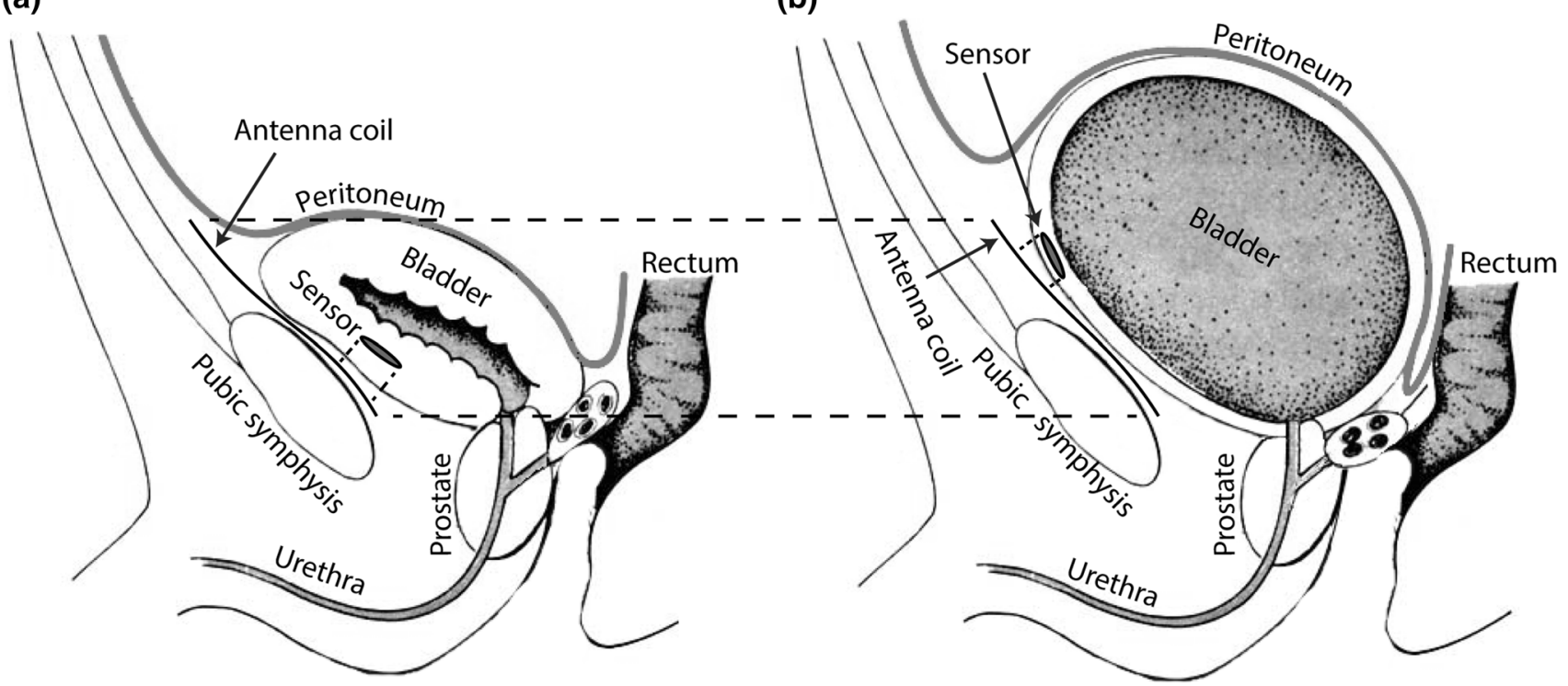

Fig. 1 Illustration of the intended placement of the proposed sensor system. a and b show lateral views of the empty and full bladder, respectively. The sensor is placed in a pouch in the middle of the bladder wall; the antenna could be anchored to the pubic symphysis. Despite movements caused by filling, the sensor is still atop the antenna coil. Modified from [23] and used by permission of the authors

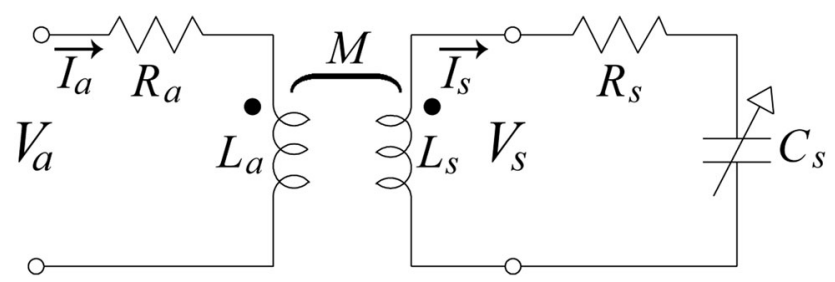

Fig. 2 Circuit diagram of the wireless sensor and antenna system

quadrature-output oscillator. Since only the frequency at which the phase dip occurs is of interest in this application, the ABB can be simplified considerably. In Fig. 3 the full $\mathrm{ABB}$ is shown (a), as well as the proposed simplified system (b). The simplified system requires only one ADC, and does not require quadrature output from the oscillator, making the system much simpler and also less power consuming. At sensor resonance, the inductive and capacitive parts of the impedance cancel, leaving only the (parasitic) resistive part. In addition, the phase of the impedance changes from $-\pi / 2$ to $+\pi / 2$. In the simplified system, this means that near sensor resonance, the sensor current increases, causing a larger modulation of the antenna current. At frequencies lower than sensor resonance, the capacitor dominates the sensor characteristic, causing the phase of the antenna to be skewed towards $-\pi / 2$. This is most pronounced just below sensor resonance (due to the larger current close to resonance). At sensor resonance, the phase of the sensor is zero, i.e. no phase modulation of the antenna coil occurs. Then, just above sensor resonance, the sensor coil becomes dominant, skewing the phase of the antenna towards $+\pi / 2$. At higher frequencies, further from the resonance, the sensor current becomes smaller, decreasing the modulation of the antenna signal. A system somewhat resembling this has previously been described by Lizon-Martinez [18], however, it was based on an operational transconductance amplifier (OTA), whereas this system uses an opamp. An advantage of the system presented here is that $R_{\text {ref }}$ can easily be adjusted to accommodate a given ADC range.

\subsection{Coil Coupling}

Coil coupling was simulated by finite element analysis (FEA) using Comsol Multiphysics v5.1 (Comsol AB, Stockholm, Sweden). Static conditions were used since coupling does not change with frequency. Similarly, intrinsic resistances do not affect coupling and were left out. In line with this, and to keep simulations simple, parasitic capacities were also neglected in the model. The antenna coil was modeled as an eight turn rectangular spiral, with outer length $40 \mathrm{~mm}$, outer width $10 \mathrm{~mm}$, and turn spacing $0.5 \mathrm{~mm}$. The sensor geometry was a square with outer side length $6 \mathrm{~mm}$, and $50 \mu \mathrm{m}$ between turns, a spacing that could be obtained easily using MEMS techniques. The coils were contained in parallel planes, with a distance between the planes ranging from $4 \mathrm{~mm}$ to $20 \mathrm{~mm}$ in $4 \mathrm{~mm}$ steps. For each distance, the sensor was translated along a grid to obtain the effect of both longitudinal and transverse translation on coupling. Thus, for each distance, a contour plot of coupling as a function of 2-D translation 
(a)
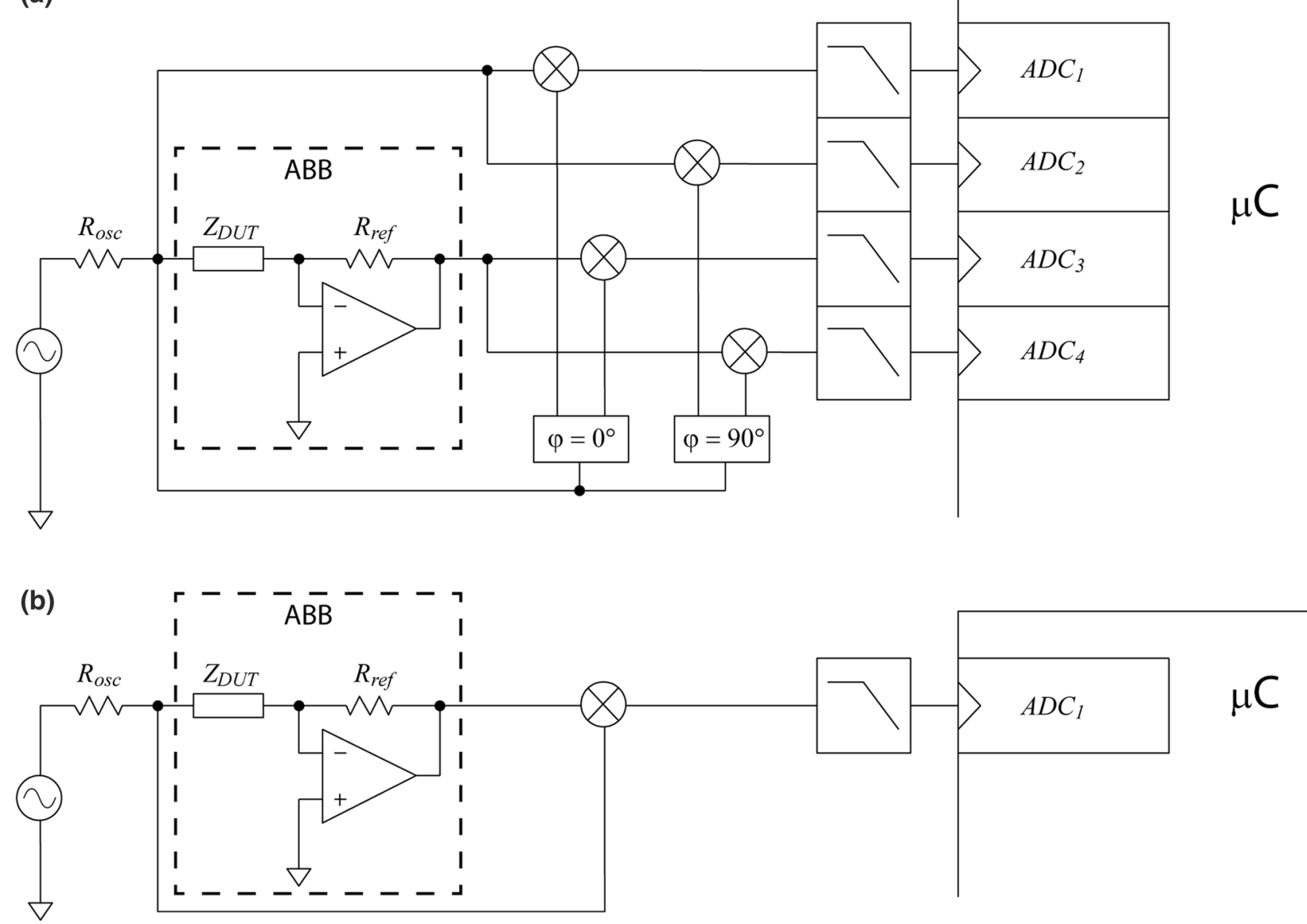

Fig. 3 Simplified system for phase monitoring. The current through $R_{\text {ref }}$ is determined by the complex impedance of $Z_{D U T}\left(Z_{D U T}\right.$ is simply the circuit shown in Fig. 2, but has been shown as a general impedance for clarity in this figure. I.e. $Z_{D U T}=V_{a} / I_{a}$ ). During a frequency sweep, the impedance phase of the sensor changes from $-\pi / 2$ to $\pi / 2$, and at resonance, the current in the sensor will attain maximal value, thereby causing most pronounced modulation of the antenna

was computed. The simulation "world" was a sphere with radius $5 \mathrm{~cm}$, filled with air.

\subsection{Full ABB System Response}

The system response was simulated using Matlab (MathWorks, Inc., Natick, MA), based on the circuit diagram presented in Fig. 3a. The opamp, resistor and multipliers were simulated as ideal components; the low pass filters were analog 1 st order passive filters with $R C=10^{-4} \mathrm{~s}$, simulated by a recursive implementation. Noise was injected in the system at the oscillator node and at the inphase and $90^{\circ}$ out-of-phase nodes. A/D conversion was not taken into account, i.e. the result is the analogue inputs to the four A/D converters shown in the figure. Thus, the simulation is of the analogue part of the system.

As can be seen from Eq. (1), the carrier modulation depends on all three parameters $R_{s}, L_{s}$ and $C_{s}$ of the sensor. For the purpose of this simulation, the values of $R_{s}$ and $C_{s}$ was estimated using standard formulae. The length of the spiral coil of the sensor is $222 \mathrm{~mm}$. The center-to-center distance of two tracks is $50 \mu \mathrm{m}$; tracks were assumed to have cross-sectional width and height of $35 \mu \mathrm{m}$, giving a track spacing of $15 \mu \mathrm{m}$. The material would typically be copper with $\rho=1.60 \cdot 10^{-8} \Omega \mathrm{m}$. Hence, the resistance of the coil is

$R=1.68 \times 10^{-8} \Omega \mathrm{m} \times \frac{0.222 \mathrm{~m}}{\left(35 \times 10^{-6} \mathrm{~m}\right)^{2}}=3 \Omega$

For the capacitor, square plates with side length $6 \mathrm{~mm}$ and gap between plates of $50 \mu \mathrm{m}$ was assumed. This gives a capacitance of

$C_{0}=\epsilon_{0} \times \frac{A}{g}=8.85 \times 10^{-12} \mathrm{Fm}^{-1} \cdot \frac{(0.006 \mathrm{~m})^{2}}{50 \times 10^{-6} \mathrm{~m}}=6.4 \mathrm{pF}$

To account for imperfections and gap variations in an actual fabrication environment, a capacity of $5 \mathrm{pF}$ was used in the simulations. Thus, the sensor parameters were 
$R_{s}=3 \Omega, L_{s}=1.4 \mu \mathrm{H}$ and $C_{s}=5 \mathrm{pF}$; the Q-factor of the simulated sensor is

$Q_{\text {sensor }}=\frac{1}{R} \cdot \sqrt{\frac{L_{s}}{C_{s}}}=176$

The sensitivity of the sensor was set to $3 \% / \mathrm{cm} \mathrm{H}_{2} \mathrm{O}$. This was found by simulation of a specific sensor layout (unpublished data by Thor Ansbæk, DTU, Denmark), and is in line with what has been reported by others. Sensitivities ranging from 0.18 to $14.7 \%$ per $\mathrm{cm} \mathrm{H}_{2} \mathrm{O}$ were reported in the literature $[9,12]$. With a pressure range of -50 to $+150 \mathrm{~cm} \mathrm{H}_{2} \mathrm{O}$ (absolute-type sensor), capacity range was 4.25 to $7.25 \mathrm{pF}$, yielding a frequency range of 50 to $65 \mathrm{MHz}$. This is also similar to the frequencies used in other studies, frequencies spanning from 13 to over $100 \mathrm{MHz}$ have been reported [11, 12, 19].

White Gaussian noise was added to the carrier signal to test the susceptibility to noise. The Gaussian property means, that with a threshold of four standard deviations, the noise is below this threshold $99.993 \%$ of the time. Conversely, this means that a phase dip modulation larger than four standard deviations will be detected correctly $99.993 \%$ of the time.

Further, the effects of $L_{s}$ and $C_{s}$ are frequency dependent. This means that the modulation magnitude changes with frequency, as illustrated in Fig. 4. In general, capacity increases with pressure, since the capacitor plates are pressed closer together. This means that both resonance

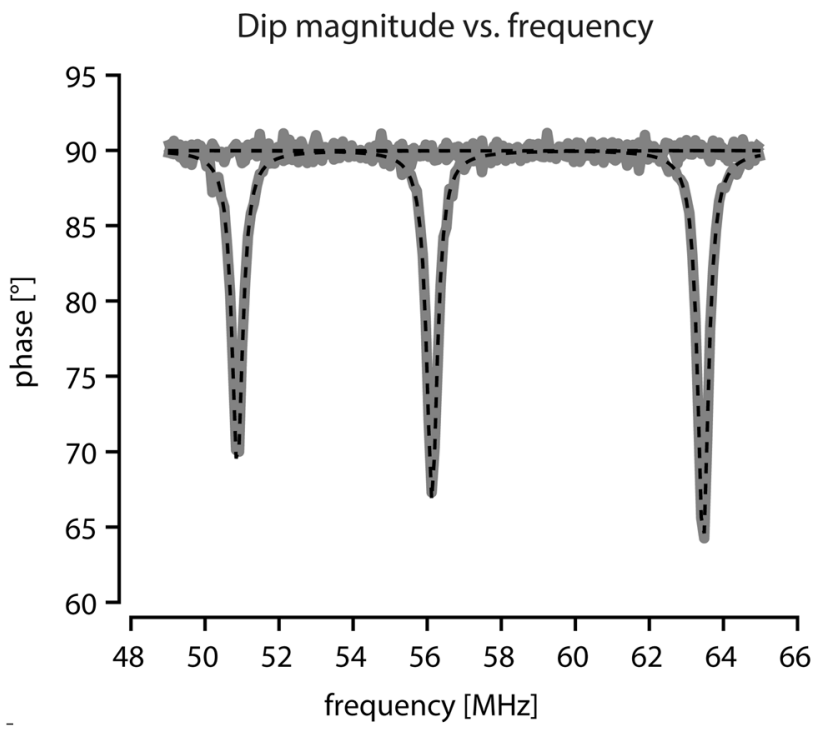

Fig. 4 Illustration of the change in dip magnitude as function of frequency for $C=4.5 \mathrm{pF}, C=5.75 \mathrm{pF}$ and $C=7.0 \mathrm{pF}$. Simulations were done with coupling $k=0.05$ and $S N R=0 \mathrm{~dB}$. The difference in dip magnitude is relative to coupling, so the absolute difference is small for low coupling coefficients. $C=7 \mathrm{pF}$ was used for all other simulations frequency and Q-factor decreases with increasing pressure. The main effect of the latter is that the phase dip also decreases in magnitude. Coupling and Q-factor can shift the dip frequency relative to the resonance frequency [13], but this effect is negligible in this study. To ensure proper functioning in the full pressure range, this is important to take into account. Hence, all simulations were done in the "worst-case scenario" with $C=7.0 \mathrm{pF}$.

In this study, a range of couplings and SNRs were examined in an exhaustive search manner, and for each combination the noise of the modulated signal, and the magnitude of the phase dip, was recorded, as illustrated in Fig. 5. Here, the theoretical phase is the phase as computed by (1), whereas the simulated measurement is computed from the ABB using standard formulae [20]. For combinations that resulted in a phase dip of smaller amplitude than the noise, the theoretical dip magnitude was used to quantify the ratio. In Fig. 5, two vertical black dashed lines indicate the boundary between dip and noise segments of the signal. These limits were computed from the theoretical phase, so they were independent of the noise level. The bell curve in the upper right corner shows the actual probability distribution of the expected noise amplitude based on the simulation.

\subsection{Simplified ABB System Simulation}

From the simplified system, an example of the response to a frequency sweep is illustrated in Fig. 6. Similar to the full system, the opamp and mixer were considered ideal, and the low-pass filter was simulated by a recursive implementation (again, $R C=10^{-4} \mathrm{~s}$ ). The oscillator had amplitude 1 , and the reference resistor was chosen to have a slightly smaller resistance than the smallest value of $Z_{D U T}$ in order to make $V_{r e f} \leq 1 \mathrm{~V}$. A value of $R_{\text {ref }}=500 \Omega$ was used. The limits of the ADC were accordingly set to 0 and $1 \mathrm{~V}$.

A simple signal detection algorithm was developed. The effect of the dip just before sensor resonance, and the peak just after, is that the slope of the curve attains its maximal value at resonance. Hence, the output from the ADC was differentiated (first order difference), and this signal was then linearly detrended. The peak of this signal denotes the resonance frequency.

As coupling decreases, the amplitude of the peak decreases as well. The ADC resolution needed to operate the sensor system, for a given coupling was determined for the noise-free case. In this simulation, there is still some noise due to quantification. The amplitude of the peak was compared to the noise amplitude, similarly to how it was done in the full ABB simulation.

Next, a typical combination from the previous simulation was chosen; a coupling of 0.005 (lower boundary of 


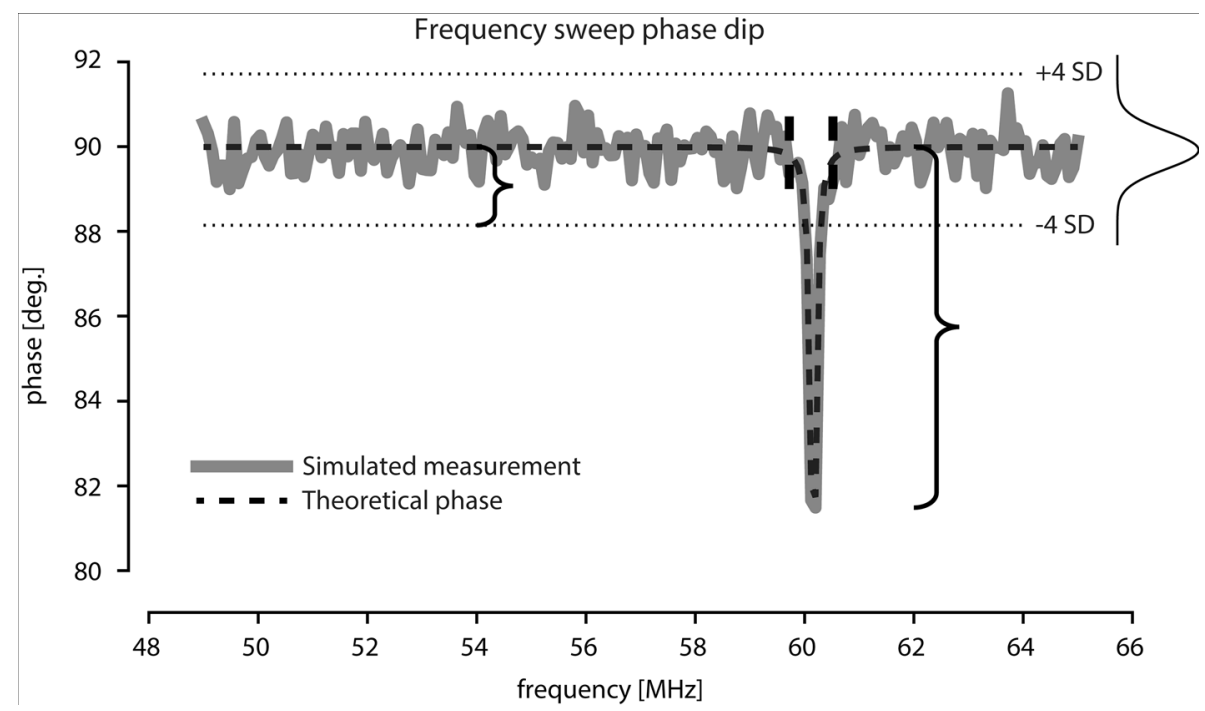

Fig. 5 A simulation of one frequency sweep. Here, coupling was 0.03 and SNR was $0 \mathrm{~dB}$. The two measurements obtained from such one simulation was the noise magnitude (calculated from the Gaussian distribution), and the dip magnitude. The vertical black dashed bars indicate onset and offset of the dip. The rest of the signal was used for computing the variance of the noise. The bell curve at the top right shows the actual expected distribution of the noise amplitude. Note that while the resonance frequency in this figure is $60 \mathrm{MHz}$ ( $5 \mathrm{pF}$ capacitor value), actual simulations were performed with a $51 \mathrm{MHz}(7 \mathrm{pF}$ capacitor value) resonance frequency

Fig. 6 Simulation of the simplified system shown in Fig. 3b. The opamp and mixer were considered ideal, the lowpass filter was simulated by a recursive implementation. Here, $\mathrm{k}=0.03$ and $\mathrm{SNR}=0 \mathrm{~dB}$.

Note that the ADC output is in steps, not voltage
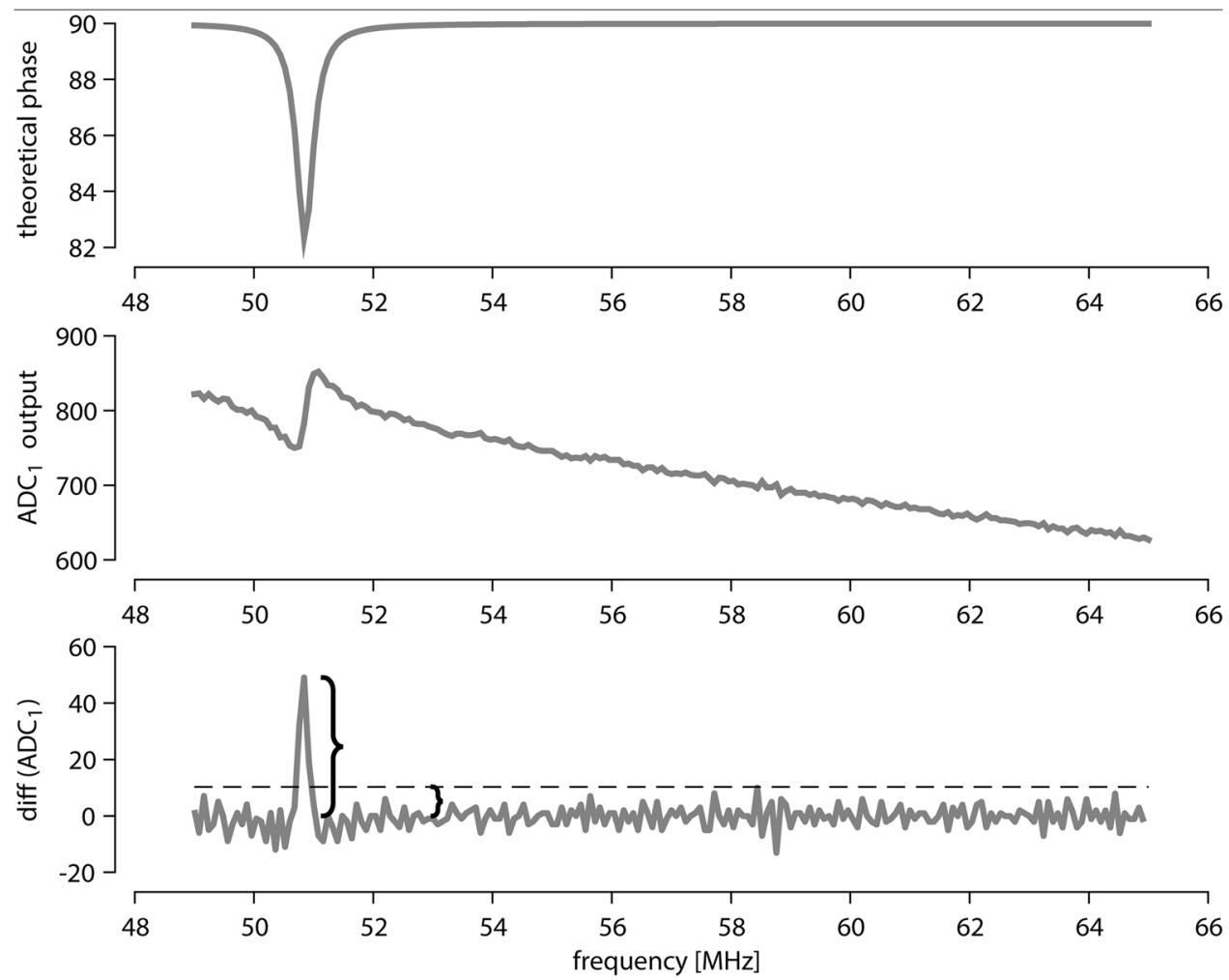

useful end), and a 14 bit ADC, which gives a peak/noise amplitude ratio of 10 . Here, noise was added to the carrier in incremental steps. A carrier signal to noise range of 0 to $40 \mathrm{~dB}$ was examined in a Monte-Carlo fashion. Hundred simulations were done for each noise level ( $\approx 3 \mathrm{~dB}$ steps), and the peak-to-noise amplitude ratio of the ADC signal, as well as the number of correct detections, were recorded. A detection was considered correct if it was within $\pm 1 \mathrm{~cm} \mathrm{H}_{2} \mathrm{O}$ of the simulated pressure. For each noise level the mean peak-to-noise ratio and the number of correct detections were reported as end points. 


\section{Results}

The results of the finite element analysis are shown in Fig. 7 as a separate contour plot for each distance. For all distances, the outermost contour corresponds to a coupling of zero. At short distances, the "field shape" corresponds well to the antenna geometry, while as distances increase, a more a more circular "field" is observed. As a measure of reliability, not only the mutual inductance, but also the antenna and sensor inductances were computed at each position. Simulated antenna inductance was $2.047 \pm 0.007 \mu \mathrm{H}$ (mean $\pm \mathrm{SD}$ ); similarly, sensor inductance was $1.422 \pm 0.003 \mu \mathrm{H}, n=210$ for both.

For the full ABB system, the dip to noise ratio are shown in Fig. 8. As this ratio varied greatly, the logarithm

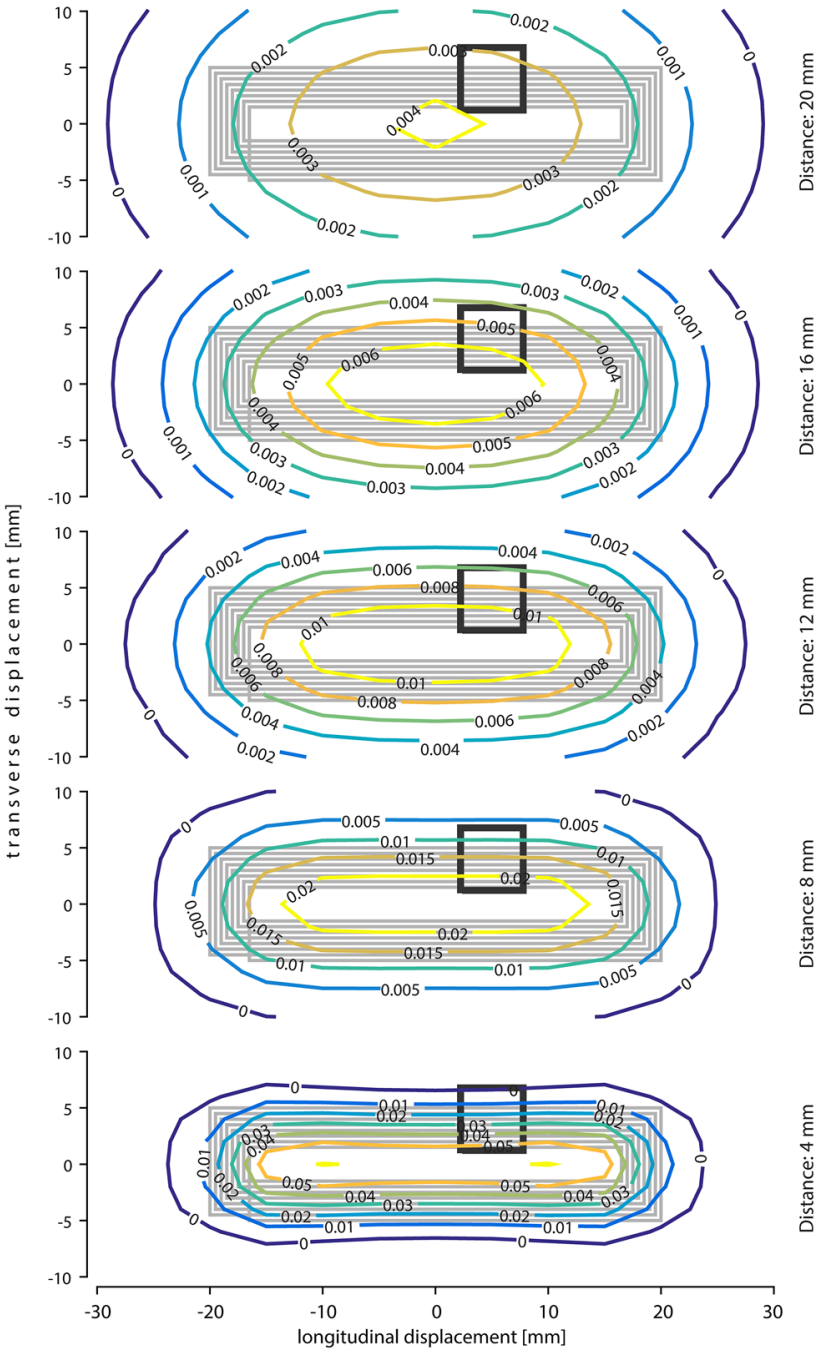

Fig. 7 Contour plots of the coupling at different positions and distances. Antenna coil is illustrated in grey, sensor coil in black. The sensor is shown translated $5 \mathrm{~mm}$ in the longitudinal direction, and $4 \mathrm{~mm}$ in the transverse direction. Coupling at this position is 0.0036 , $0.0057,0.0093,0.015$ and 0.024 , respectively, for distances decreasing from $20 \mathrm{~mm}$ to $4 \mathrm{~mm}$

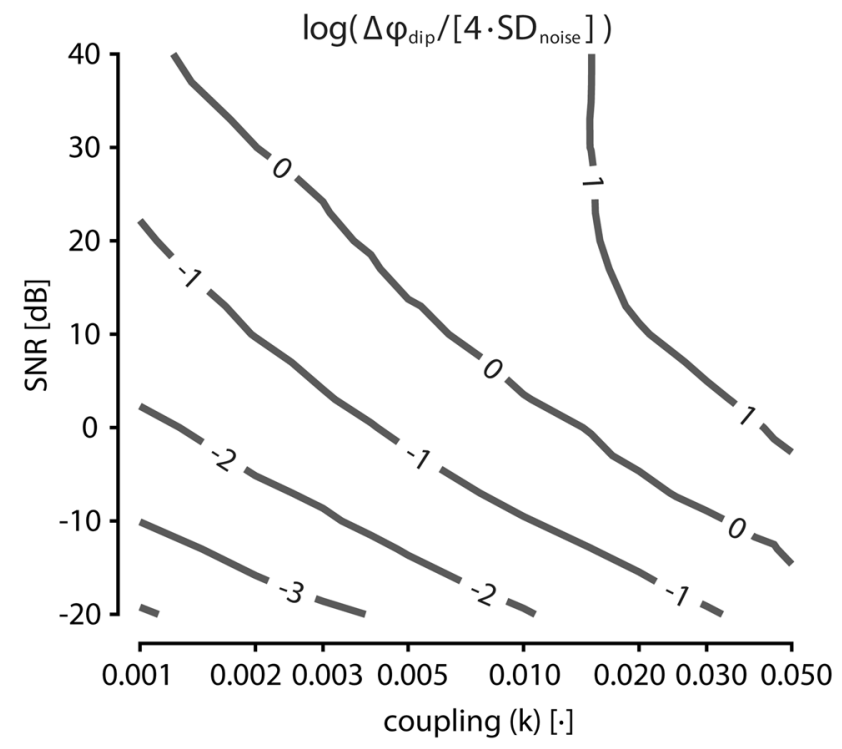

Fig. 8 Dip magnitude as function of coupling and SNR

of the ratio is shown. Hence, the zero contour-line in Fig. 8 indicates the boundary where dip magnitude and noise amplitude are equal. It is seen that for a large coupling, e.g. $k=0.05$, a large amount of noise can be tolerated ( $S N R=-10 \mathrm{~dB})$, whereas for a small coupling, e.g. $k=0.005$, an $S N R=13 \mathrm{~dB}$ or better is required for proper operation.

For the simplified system, the required ADC resolution in the noise-free case is shown in Fig. 9, as the zero contour-line (the plot shows $\log _{10}$ of the peak-to-noise ratio). From this is also seen how much either the coupling or the number of bits needs to be increased to obtain a certain noise margin.

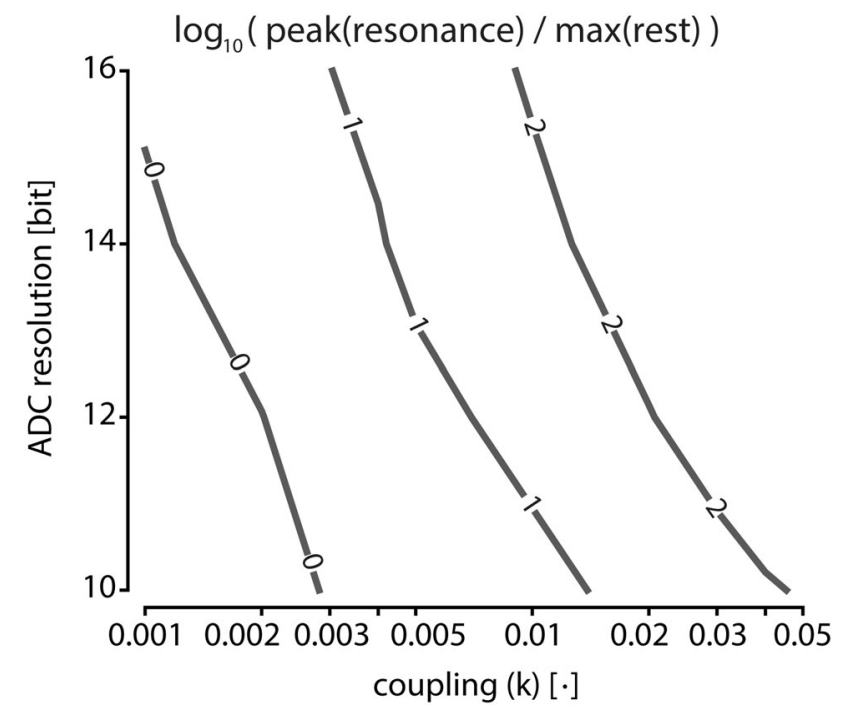

Fig. 9 Required ADC resolution to operate the sensor system using the simplified ABB topology 
Finally, the results of the Monte-Carlo simulation are reported in Table 1.

\section{Discussion}

The aim of this work was to characterize systems for operating wireless passive LC-tank sensors, and in particular, to evaluate, by simulations, a specific proposed system for bladder monitoring. The design for the bladder monitoring system was based upon an expected short distance between antenna and sensor. The desire for a wireless system, even given this short operating range, is because the bladder constantly undergoes filling and voiding cycles, causing repeated movement. If connecting wires for a bladder sensor become fixed to tissue, e.g. by connective tissue growth, this could cause sensor migration [8]. It was not investigated if it is possible to fix the antenna in the position described in Fig. 1, nor was the amount of fatty tissue around the bladder in typical wheel-chair bound patients, investigated. Therefore, distances between $4 \mathrm{~mm}$ and $20 \mathrm{~mm}$ were simulated. At short distances, the desired behavior, a "coupling field" shaped similarly to the antenna, was obtained, causing relatively high and nearly constant coupling for the intended range of motion. At longer distances, coupling rapidly decreased.

There was a very high degree of consistency between simulations, as seen by the very small standard deviation of the computed coil values across simulations. Thus, although the absolute values may be somewhat off, the qualitative features of the coupling patterns described are very likely correct.

The contour plots shown in Fig. 7 do not show negative coupling coefficients. While the system operates equally well for positive or negative coupling coefficients, the sensor would have to cross the zero-coupling zone during this transition. This would mean a functional dead space where bladder pressure could not be monitored. A system with an array of antenna coils could be envisioned, where the response magnitude was monitored, and when this magnitude falls below a certain threshold, the next coil in the array could be used to continue operation. This was not considered in this work.

The coupling coefficients were simulated under ideal conditions. Air is a non-conducting (lossless) medium, and

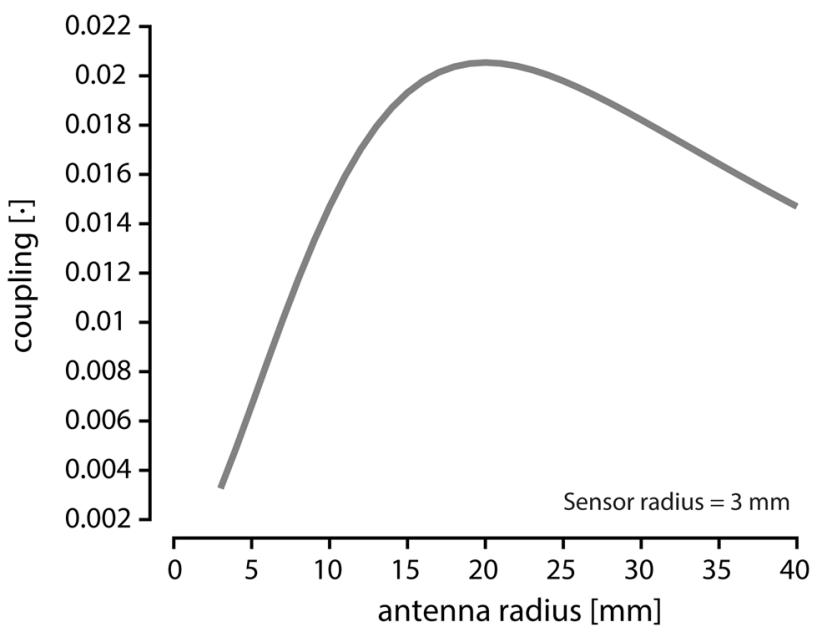

Fig. 10 Approximated coupling as a function of antenna radius. The model assumes two circular co-axial wire loops. The loops are spaced $20 \mathrm{~mm}$ apart, and radius of the smaller loop (corresponding to sensor coil) has radius $3 \mathrm{~mm}$

there were no obstacles interfering with the field distribution. Specifically, the capacitor plates were not considered in these simulations. Depending on manufacturing technique, the capacitor could be placed on top of the coil, besides the coil, or the coil(s) could act as one or both of the capacitor plates. Generally, the capacitor plates will influence the coupling in a negative direction.

The coupling of two single-turn co-axial circular wire loops, can be approximated by

$k\left(r_{1}, r_{2}, x\right) \approx \frac{r_{1}^{2} \cdot r_{2}^{2}}{\sqrt{r_{1} \cdot r_{2}}\left(\sqrt{x^{2}+r_{2}^{2}}\right)^{3}}$,

where $r_{1}, r_{2}$ are the radii $\left(r_{1}<r_{2}\right)$, and $x$ is the distance [21].

It should thus be possible to obtain a coupling of 0.02 , with a $\varnothing 40 \mathrm{~mm}$ antenna (Fig. 10). While this is not directly comparable with square spirals, a similar coupling could be expected. Square spiral antenna coils (side length $40 \mathrm{~mm}$ ) were simulated and compared to the proposed system. At a short distance $(4 \mathrm{~mm})$ the coupling coefficient was smaller than that of the proposed system $(0.03$ vs. $0.05)$, however at a long distance $(20 \mathrm{~mm})$, coupling was generally larger (0.005-0.007 vs. 0.002-0.004) than for the proposed system. At a long distance, the spread of the field causes a local coupling minimum for zero translation, i.e.

Table 1 Results from the Monte-Carlo simulation of the simplified system given $k=0.005$ and a 14 bit ADC. First row shows the carrier SNR, second row shows the mean max peak-to-max noise amplitude ratio, and last row the number of correct detections $(n=100$ for each SNR)

\begin{tabular}{|c|c|c|c|c|c|c|c|c|c|c|c|c|c|}
\hline SNR (dB) & 0 & 3 & 7 & 10 & 13 & 17 & 20 & 23 & 27 & 30 & 33 & 37 & 40 \\
\hline Peak/noise & 0.42 & 0.50 & 0.66 & 0.71 & 0.81 & 1.11 & 1.53 & 2.12 & 3.04 & 4.10 & 5.33 & 6.96 & 7.82 \\
\hline \#Correct & 4 & 3 & 13 & 20 & 23 & 52 & 92 & 99 & 100 & 100 & 100 & 100 & 100 \\
\hline
\end{tabular}


when the two coils have aligned centers. This would disrupt sensor operation when placed in this location. Also, the coupling at $20 \mathrm{~mm}$ distance is far below what was predicted by the approximation formula. To verify the results obtained by the simulation, the simulated inductances were compared with known estimation formulae. From the simulations, the inductances of the sensor and antenna were $L_{s}=1.423 \pm 0.003 \mu \mathrm{H}$ and $L_{a}=7.593 \pm$ $0.014 \mu \mathrm{H}$, respectively. Using the current sheet approximation formula of Mohan et al. [22], the computed inductances were $L_{s, g m d}=1.44 \mu \mathrm{H}$ and $L_{a, g m d}=7.98 \mu \mathrm{H}$, respectively. This indicates that the simulation results are correct, and that the coupling approximation formula (3), for some reason, does not work well in this setting.

For the simulations of the analogue part of the $\mathrm{ABB}$, the last step is to low-pass filter the signal. This means that the added white Gaussian noise becomes colored, and that the distribution generally becomes more platykurtic. Since a platykurtic distribution is less prone to outliers than a normal distribution, this only strengthens the reliability of using four standard deviations as threshold.

In this study, ADCs with resolution from 10 to 16 bits were considered. The necessary resolution depends on the analogue circuitry feeding the ADC, and the dynamic range of the signal. In the case of the simplified system, the dynamic range is rather low. Given the described choice of $R_{\text {ref }}$, the signal typically attains values from 0.6 to $0.9 \mathrm{~V}$ or less, as seen in Fig. 6. By subtracting the offset of the signal, an additional bit could be added to the resolution of the signal. Also, even though the oscillator is operating at high frequencies, the ADC needs to operate only at a low frequency of $1000 \mathrm{~Hz}$. This allows for 200 discrete frequencies (200 $\mathrm{cm} \mathrm{H}_{2} \mathrm{O}$ resolution of the sensor), and five readings per second. This relaxes the requirements to the ADC considerably, and allows for a long hold time making it possible to use high resolution but slower $\Sigma \Delta$-type ADCs.

The findings of this study were not verified by laboratory measurements. The most critical issues for implementing the system is the placement of the antenna, the distance between sensor and antenna, and movement path. This will elucidate if a coupling of e.g. at least 0.005 can be maintained during normal operation, and hence whether the system is feasible. If so, it will certainly be possible to fulfill the requirements to the signal processing part of the system. Further studies should address this issue.

In conclusion, it was shown that the presented coil design provides nearly constant coupling at close distances during linear movement. For distances up to $12 \mathrm{~mm}$, an adequate coupling coefficient could be maintained. The combination of signal to noise ratio and coupling coefficient determines the detection limit of the system. For coupling coefficients above 0.005 , the requirements to the system are such that it is still feasible. By combining these results, the system can operate at coil distances up to $12 \mathrm{~mm}$, giving a coupling coefficient of at least 0.005 . In this case a 14 bit ADC gives a baseline peak-to-noise ratio of 10 ADC steps. Noise can occur in the system until the SNR is $27 \mathrm{~dB}$ (or better), at which point correct detections are still expected to occur close to $100 \%$ of the time.

\section{References}

1. Hansen, J., Media, S., Nohr, M., Biering-Sørensen, F., Rijkhoff, N. J. M., \& Sinkjaer, T. (2005). Treatment of neurogenic detrusor overactivity in spinal cord injured patients by conditional electrical stimulation. Journal of Urology, 173, 2035-2039.

2. Melgaard, J., \& Rijkhoff, N. J. (2011). Detecting the onset of urinary bladder contractions using an implantable pressure sensor. IEEE Transactions on Neural Systems and Rehabilitation Engineering, 19, 700-708.

3. Takayama, K., Takei, M., Soejima, T., \& Kumazawa, J. (1987). Continuous monitoring of bladder pressure in dogs in a completely physiological state. British Journal of Urology, 60, 428-432.

4. Mills, I. W., Noble, J. G., \& Brading, A. F. (2000). Radiotelemetered cystometry in pigs: Validation and comparison of natural filling versus diuresis cystometry. The Journal of Urology, 164, 1745-1750.

5. Gerber, M. T. (2007). Transmembrane sensing device for sensing bladder condition. US2007/0027494 A1.

6. Shaw, G. L., Choong, S. K., \& Fry, C. (2005). Encrustation of biomaterials in the urinary tract. Urological Research, 33, 17-22.

7. Stickler, D. J. (2012). Surface coatings in urology. In M. Driver (Ed.), Coatings for biomedical applications (pp. 304-335). Cambridge: Woodhead Publishing.

8. Koldewijn, E. L., van Kerrebroeck, P. E. V., Schaafsma, E., Wijkstra, H., Debruyne, F. M. J., \& Brindley, G. (1994). Bladder pressure sensors in an animal model. Journal of Urology, 151, 1379-1384.

9. Park, E. C., Yoon, J., \& Yoon, E. (1998). Hermetically sealed inductor-capacitor (LC) resonator for remote pressure monitoring. Japanese Journal of Applied Physics, 37, 7124-7128.

10. Puers, R., Vandevoorde, G., \& de Bruyker, D. (2000). Electrodeposited copper inductors for intraocular pressure telemetry. Journal of Micromechanics and Microengineering, 10, 124-129.

11. Akar, O., Akin, T., \& Najafi, K. (2001). A wireless batch sealed absolute capacitive pressure sensor. Sensors and Actuators A, 95, 29-38.

12. DeHennis, A., \& Wise, K. D. (2002). A double-sided single-chip wireless pressure sensor. In The fifteenth IEEE international conference on micro electro mechanical systems (pp. 252-255).

13. Fonseca, M. A., English, J. M., von Arx, M., \& Allen, M. G. (2002). Wireless micromachined ceramic pressure sensor for high-temperature applications. Journal of Microelectromechanical Systems, 11, 337-343.

14. Chen, P. J., Rodger, D. C., Saati, S., Humayun, M. S., \& Tai, Y. C. (2008). Implantable parylene-based wireless intraocular pressure sensor. In IEEE 21st international conference on micro electro mechanical systems (MEMS) (pp. 58-61).

15. Lotz, H. T., Remeijer, P., van Herk, M., Lebesque, J. V., de Bois, J. A., Zijp, L. J., et al. (2004). A model to predict bladder shapes from changes in bladder and rectal filling. Medical Physics, 31(6), 1415-1423. doi:10.1118/1.1738961. 
16. Fokdal, L., Honoré, H., Høyer, M., Meldgaard, P., Fode, K., \& von der Maase, H. (2004). Impact of changes in bladder and rectal filling volume on organ motion and dose distribution of the bladder in radiotherapy for urinary bladder cancer. International Journal of Radiation Oncology Biology Physics, 59, 436-444.

17. Black, J. (1992). Biological performance of materials: Fundamentals of biocompatibility. New York: Dekker.

18. Lizon-Martinez, S., Giannetti, R., Rodriguez-Marrero, J. L., \& Tellini, B. (2005). Design of a system for continuous intraocular pressure monitoring. IEEE Transactions on Instrumentation and Measurement, 54, 1534-1540.

19. Collins, C. C. (1967). Miniature passive pressure transensor for implanting in the eye. In IEEE Transactions on Biomedical Engineering, vol. BME-14, pp. 74-83.
20. Hall, H. P. (1980). Method of and apparatus for automatic measurement of impedance or other parameters with microprocessor calculation techniques, 04/01.

21. Finkenzeller, K. (2010). RFID handbook: Fundamentals and applications in contactless smart cards, radio frequency identification and near-field communication. Chichester: John Wiley Sons Ltd.

22. Mohan, S. S., Hershenson, M. D. M., Boyd, S. P., \& Lee, T. H. (1999). Simple accurate expressions for planar spiral inductances. IEEE Journal of Solid-State Circuits, 34, 1419-1424.

23. O'Rahilly, R., Müller, F., Carpenter, S., \& Swenson, R. (2008). Basic human anatomy: A regional study of human structure. Retrieved from http://www.dartmouth.edu/ humananatomy/. 